\title{
Estimating the risk of SARS-CoV-2 transmission to pediatric anesthesiologists: a microsimulation model
}

\author{
Kazuyoshi Aoyama, MD, PhD (D) Anna Heath, PhD • Alan Yang, MSc • \\ Jason T. Maynes, PhD, MD • Guy Petroz, MD • James Robertson, MD • \\ Conor Mc Donnell, MB, MD • Russanthy Velummailum, MPH • Elizabeth Bond, MPH • \\ Petros Pechlivanoglou, PhD
}

Received: 17 July 2020/Revised: 19 July 2020 / Accepted: 19 July 2020/Published online: 27 July 2020

(C) Canadian Anesthesiologists' Society 2020

\section{To the Editor,}

Anesthesiologists are at high risk of aerosol-transmitted infection by severe acute respiratory syndrome coronavirus-2 (SARS-CoV-2) during their airway management of patients with coronavirus disease (COVID-19). Adding to this, many patients with COVID19 are asymptomatic, especially children, ${ }^{1}$ and aerosol transmission can occur despite the use of full personal protective equipment. ${ }^{2}$ Most acute-care hospitals in North America suspended elective surgeries soon after the pandemic was declared to reduce the spread of SARSCoV-2 in patients and healthcare workers (HCWs), as well as to conserve hospital resources for the anticipated surge of COVID-19 patients. It remains unknown how cancelling elective surgeries initially impacted, and will continue to impact, the risk of SARS-CoV-2 transmission from a patient to anesthesiologists, or the use of hospital resources. Thus, we created an open source, online

\footnotetext{
K. Aoyama, MD, PhD ( $\varangle)$.

Department of Anesthesia and Pain Medicine, The Hospital for Sick Children, Toronto, ON, Canada

e-mail: kazu.aoyama@utoronto.ca
}

Program in Child Health Evaluative Sciences, SickKids Research Institute, Toronto, ON, Canada

\author{
A. Heath, PhD \\ Program in Child Health Evaluative Sciences, SickKids \\ Research Institute, Toronto, ON, Canada
}

Biostatistics Division Dalla Lana School of Public Health, University of Toronto, Toronto, ON, Canada

A. Yang, MSc $\cdot$ R. Velummailum, MPH $\cdot$ E. Bond, MPH Program in Child Health Evaluative Sciences, SickKids Research Institute, Toronto, ON, Canada microsimulation model (https://pechlilab.shinyapps.io/ COMPS-GTA/) to estimate the effect of cancelling elective surgeries on the risk of SARS-CoV-2 transmission from a pediatric patient to anesthesiologists during the COVID-19 pandemic.

The Hospital for Sick Children Research Ethics Board approved this study; no.1000070090). The microsimulation model allowed us to explore pivotal factors while resuming elective surgeries. The microsimulation model is based on principles and methods previously described. ${ }^{3,4}$ A userfriendly interface enhances access to the model and allows customized use by other hospitals through the adjustment of parameters for local settings. Specific assumptions and input parameters used in the model are described within the interface, which we will continue to update for more accuracy and validation as additional evidence becomes available. Pre-defined inputs and assumptions derived from our institution and the existing literature were used to

J. T. Maynes, PhD, MD

Department of Anesthesia and Pain Medicine, The Hospital for Sick Children, Toronto, ON, Canada

Program in Molecular Medicine, SickKids Research Institute, Toronto, ON, Canada

G. Petroz, MD - J. Robertson, MD - C. Mc Donnell, MB, MD Department of Anesthesia and Pain Medicine, The Hospital for Sick Children, Toronto, ON, Canada

P. Pechlivanoglou, $\mathrm{PhD}$

Program in Child Health Evaluative Sciences, SickKids Research Institute, Toronto, ON, Canada

Institute for Health, Policy, Management and Evaluation, University of Toronto, Toronto, ON, Canada 
Probability of at least one anesthesiologist being infected

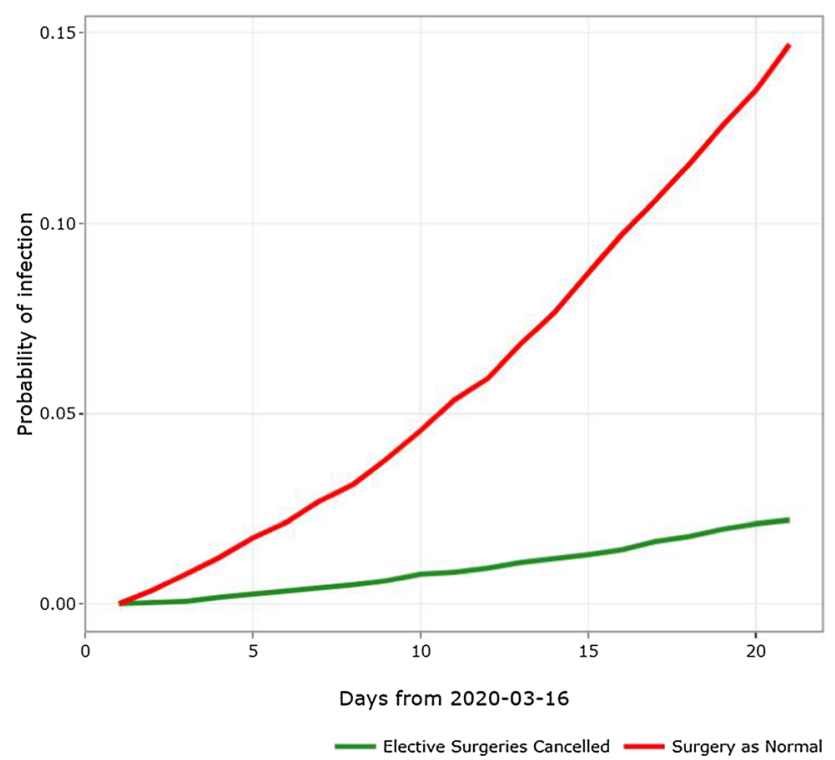

FIGURE Microsimulation of the cumulative incidence of severe acute respiratory syndrome-associated coronavirus- 2 transmission to an anesthesiologist (model data are from 16 March to 5 April 2020). The solid red line denotes the scenario if elective surgeries were being continued with the solid green line denoting a scenario where elective surgeries were cancelled.

produce estimates. Our scenarios draw upon the daily cases of COVID-19 infections in the Greater Toronto Area, Canada, derived from Government of Ontario (https:// covid-19.ontario.ca/index.html). ${ }^{\text {A }}$ Through the interface, users can define inputs such as surgical statistics, including percent change of surgical caseload, and constraint on the number of available N95 respirators to model expected resource utilization at the individual hospital level, community level, or provincial level.

Beginning on 16 March 2020, our quaternary-care children's hospital performed only emergent and urgent surgeries (e.g., cancer surgery), including 236 cases during the first three weeks after the pandemic was declared. This is compared with a total of 1,578 similar cases during the same three-week period in 2019. We estimated that cancelling elective surgeries during those three weeks reduced the cumulative incidence of SARS-CoV-2 transmission to an anesthesiologist by more than six times $(2.1 \%$ with cancellation compared with $13.5 \%$ without cancellation) (Figure).

The microsimulation model applies only to the pediatric population since the incidence of symptoms in children who are COVID-19 positive appears to be different from that in adults. ${ }^{1}$ Instead of reuse of N95 respirators,

\footnotetext{
$\overline{{ }^{A} \text { Government }}$ of Ontario. Think you might have COVID-19 (coronavirus)? Available from URL: https://covid-19.ontario.ca/ index.html (accessed July 2020)
}

extended use of N95 respirators was assumed (i.e., an anesthesiologist wearing the same N95 respirator for all cases during their shift). We assumed the use of regular surgical masks if the stock of N95 respirators was depleted. ${ }^{5}$ Although we considered aerosol transmission and environmental contamination of SARS-CoV-2 in the model, we did not account for transmission risks among HCWs in operating rooms, which is our future work. Instead of adjusting the duration of each surgery, we employed a fixed risk of transmission to an anesthesiologist conducting an airway management. The user can tune the transmission risk in the model when new data emerge. We incorporated our hospital's preoperative screening strategy of SARS-CoV-2 into the model with diagnostic accuracy.

The first priority in a circumstance of unprecedented stress such as the COVID-19 pandemic is to maintain HCW safety so healthcare systems can provide necessary patient care. This comes with the cost of possibly extending surgical waiting time beyond what is considered safe, which may then result in an elective surgery becoming emergent. We hope this interface will aid clinicians, administrators, and policymakers in the decision-making process involving resuming elective surgeries to optimize patient care during the COVID-19 pandemic. After external validation of the model, the interface could be utilized for a possible second wave of the COVID-19 pandemic and a similar situation in a future pandemic.

\section{Disclosures None.}

Funding statement Anna Heath was funded through an Innovative Clinical Trials Multi-year Grant from the Canadian Institutes of Health Research (funding reference number MYG-151207; 20172020).

Editorial responsibility This submission was handled by Dr. Hilary P. Grocott, Editor-in-Chief, Canadian Journal of Anesthesia.

Ethical approval The current study was approved by the Research Ethics Board of the Hospital for Sick Children on 16 April 2020, REB number: 1000070090.

\section{References}

1. Lu X, Zhang $L, D u H$, et al. SARS-CoV-2 Infection in children. $\mathrm{N}$ Engl J Med 2020; 382: 1663-5.

2. Fowler RA, Guest CB, Lapinsky SE, et al. Transmission of severe acute respiratory syndrome during intubation and mechanical ventilation. Am J Respir Crit Care Med 2004; 169: 1198-202.

3. Siebert $U$, Alagoz $O$, Bayoumi AM, et al. State-transition modeling: a report of the ISPOR-SMDM Modeling Good Research Practices Task Force-3. Value Health 2012; 15: 812-20.

4. Krijkamp EM, Alarid-Escudero F, Enns EA, Jalal HJ, Hunink MG, Pechlivanoglou $P$. Microsimulation modeling for health decision sciences using R: a tutorial. Med Decis Making 2018; 38: 400-22. 
5. Sun $M$, Zhang $J$, Zhang $W$, et al. Predictive factors of transmission during endotracheal intubation for coronavirus disease 2019 (COVID-19). Lancet Preprint 2020; DOI: https://doi.org/10.2139/ ssrn.3557990.
Publisher's Note Springer Nature remains neutral with regard to jurisdictional claims in published maps and institutional affiliations. 